\title{
An Efficient MBMS Content Delivery Scheme over the HSDPA Network
}

\author{
Ahsan Chaudhry, Jamil Y. Khan \\ School of ELEC Engg. and COMP Science, The University of Newcastle, Australia \\ ahsan.chaudhry@studentmail.newcastle.edu.au,Jamil.khan@newcastle.edu.au
}

\begin{abstract}
Multimedia Broadcast and Multicast Service (MBMS) specification was introduced in the release 6 of the $3 G P P$ standard to deliver multimedia content to a large group of users in the UMTS network. With the MBMS system generally the same content is transmitted to multiple users in a unidirectional fashion, typically by multiple base stations to improve the coverage over a large geographical area. From the radio network point of view the standard supports the point-to-point (p-t-p) and the point-to-multipoint (p-t-m) transmission modes. In this paper we propose a group based $p-t-m$ MBMS scheme utilising the scalable video coding techniques to improve overall QoS of multimedia services. The group based MBMS scheme has been proposed to support moderate to high date rate multicasting services over the HSDPA link. The proposed scheme has been simulated using an OPNET simulation model. Initial results show that the proposed scheme could offer higher data and improved QoS for MBMS users.
\end{abstract}

\section{Introduction}

The Multimedia Broadcast Multicast Service $(M B M S)$ standard which was proposed in the release 6 of the 3GPP standard can support efficient multicasting and broadcasting services over the UMTS network [1].The MBMS standard is overlayed on the existing UMTS network to reduce the cost of the system development. Hence, it is possible for MBMS service providers to take advantage of the increasing data rate of the HSPA (High Speed Packet Access) system to introduce diverse and high quality multimedia contents. The standard supports both point-to-point (p-t-p) and point-to-multipoint ( $p-t-m)$ transmission modes. The p-t-p mode is more suitable for small number of users whereas the $\mathrm{p}-\mathrm{t}-\mathrm{m}$ mode is more suitable for large number of users. Since, the MBMS is overlayed on top of the UMTS network the network can automatically take advantage of the network point to point channels to carry service level feedback from users. The quality of MBMS services in a network will depend on radio conditions and cellular network design. To support MBMS services macro diversity was proposed where combining transmissions of the same content from multiple base stations provide a significant diversity gain. The macro diversity was proposed to avoid relaying feedback from users which would consume a large amount of bandwidth on the uplink [2]. Such approach could improve the coverage area and offer a reduction of 4-6 $\mathrm{dB}$ transmission power compared to a single reception only. However, the drawback of the system is use of higher radio resources because the same content is transmitted via multiple base stations. In this paper we propose an improved MBMS QoS and service coverage technique based on single Node-B solution. Using the approach multicast group receivers are separated into groups where the scalable video coders are used to receive multimedia contents. The proposed scheme is implemented on the High Speed Downlink Packet Access (HSDPA) standard [3].

To develop the proposed scheme we assume that future multimedia content can be generated using scalable video coders where a significant amount of bandwidth is consumed by video contents. In a wireless network to receive high quality multimedia content it is necessary to have a high data rate link. High data rate links can only be allocated to users if the link can maintain sufficient SNR value. Maintaining high SNR throughout a cell is extremely difficult and this factor is one of the bottlenecks of wireless systems. In this work we examined a HSDPA based MBMS system where multimedia services could be provided without use of macro diversity. We combine the concept of group based multicasting and scalable video coding technique to improve the overall QoS of a MBMS system. A scalable video coder can create multilayered coded video stream to send video contents by generating an array of data packets [4]. The selectable nature of the codec will allow receivers to select number of layers it wants to receive. In a wireless network a receiver can select the number of video layers based on their available transmission link rate. Without the macro diversity it is well known that data rates towards cell edges will always be lower compared to data rates around the vicinity of a base station. On the other hand if multicast user group is formed by combining users from different locations of a cell using standard video coders to receive multimedia content then the variability of the transmission link quality will constrain the achievable data rate of the user. If users select their video coding rate based on their link qualities then a number of multicast groups can be formed where users can select their video coding 
rates based on their transmission link qualities and can improve the overall QoS of the system.

Our proposed group based multicasting scheme has been developed on the HSDPA standard. As one of the initial steps of the 3GPP Long Term Evolution (LTE) plan, the HSDPA standard has been introduced to significantly improve downlink capacity over next few years. The HSDPA architecture was initially introduced in the 3GPP release 5. The HSDPA channel uses a short $2 \mathrm{~ms}$ transmission time interval (TTI), and uses variable coding and modulation techniques to achieve high data rate. In addition to above techniques the node-B based fast scheduling and fast physical layer hybrid automatic repeat request (H-ARQ) techniques are used in the HSDPA standard. The Standard supports a fast channel-dependent scheduling to allocate shared channel at any particular moment considering the instantaneous radio channel conditions. This makes HSDPA an ideal platform to implement future MBMS services.

The paper is organised as follows. Section 2 reviews several relevant papers on HSDPA based MBMS services. Section 3 describes our proposed group based multicast transmission scheme. Simulation model and parameters are described in the section 4. Simulation results are presented in the section 5 and paper is concluded in section 6 .

\section{HSDPA Based MBMS}

With the advancement of the HSDPA standard, research community have shown a lot of interest in exploring its adaptive high data rate capability to improve system throughput and QoS for different Real Time and Non-Real Time Applications. MBMS is considered to be one of the services which could generate significant revenues for mobile operators and therefore has been investigated for improving user perceived QoS of multimedia applications using the HSDPA standard. The MBMS can be delivered using either $\mathrm{p}-\mathrm{t}-\mathrm{p}$ or $\mathrm{p}-\mathrm{t}-\mathrm{m}$ mode of transmission. Capability for transmitting MBMS over HSDPA in p-t-p mode has been studied in the reference [5] and authors have presented a fair channel-dependent scheduler. The paper concluded that for strict delay and jitter requirements of streaming video traffic, HSDPA can support up to 15 users per tri-sector cell, serving more users decreases capacity and increases the delay. It is also proposed that the HSDPA could be used as an additional p-t-p repair mechanism for recovering lost packets instead of standalone operation to support MBMS services. In the reference [6] different multi-resolution schemes have been proposed to improve the MBMS performance. One of the proposals is the use of HSDPA as a multi-resolution technique to achieve different data rates based on the reported feedback. Results show improvement in average system throughput and authors have found it to be "an interesting option". However, the technique only considers HSDPA p-t-p mode and therefore not being able to serve large number of users. The reference [7] considered the HSDPA p-t-m mode and looked into the coverage of MBMS with different fixed number of retransmissions, and Modulation and Coding Sets (MCS). Results showed that for all combinations 1 to 4 retransmissions are required to achieve above $95 \%$ coverage. Since the retransmissions uses additional radio resources, the goodput decreases with the number of retransmissions. Authors also looked into the adaptive retransmissions issues based on UE feedbacks and showed the improvement of transmission resource utilisation by up to $70 \%$ is possible. Although not directly mentioned but it is still implied that any number of retransmission including the adaptive feedback mechanism reduces the overall goodput.

\section{Group Based Multicast Transmission Scheme}

We propose a group based transmission scheme to support MBMS services over the HSDPA network. An HSDPA connection adaptively set its transmission rate based on the Channel Quality Indicator (CQI) value obtained via the user feedback. To serve large population of multimedia users in a cell it is unrealistic to expect that all users can receive same data rate and hence it is realistic to expect that received data rate for different users will be different. In order to implement acceptable multimedia services we propose a group based transmission scheme which takes advantage of scalable video coding technique. We have chosen the scalable video coding extension of H.264/AVC Standard for video coding due to its efficient video compression technique. The encoder generates the coded stream into one Base layer and multiple Enhancement layers. We will be using temporal scalability which maintains the same spatial resolution with different frame rate in all the layers. At the Node-B, Base and multiple Enhancement video layers are mapped onto HSDPA connections based on their reported CQIs. The Node-B transmits video to different UEs which may contain only the base layer or base layer plus one or two enhancement layers as shown in table I. UEs will receive the appropriate video content based on their received $\mathrm{CQI}$. For example, if an UE is receiving a CQI value of 5 then it will receive the content of group 2 as shown in the table I. If the CQI of an UE changes considerably then the UE will move to a different group and receive the appropriate content. As for example, if the CQI of an UE decreases from 5 to 3 then that UE will move from group 2 to 1 . 
TABLE I. Scalable Video mapping to HSDPA MCS

\begin{tabular}{|c|c|c|c|c|c|}
\hline \multicolumn{5}{|c|}{ Scalable Encoded Video } & \multicolumn{2}{c|}{ HSDPA MCS } \\
\hline $\begin{array}{c}\text { Gr } \\
\text { ou } \\
\text { p }\end{array}$ & Layer & $\begin{array}{c}\text { Resolution @ } \\
\text { frame Rate } \\
\text { (fps) }\end{array}$ & $\begin{array}{c}\text { Data } \\
\text { Rate } \\
\text { Kbps }\end{array}$ & CQI & $\begin{array}{c}\text { Data } \\
\text { Rate } \\
\text { Kbps }\end{array}$ \\
\hline 1 & Base & $352 \times 288 @ 30$ & 75.5 & 2 & 86.5 \\
\hline 2 & Base+E1 & $352 \times 288 @ 30$ & 156 & 4 & 158 \\
\hline 3 & $\begin{array}{c}\text { Base+E1+ } \\
\text { E2 }\end{array}$ & $352 \times 288 @ 30$ & 396 & 8 & 396 \\
& & & & \\
\hline
\end{tabular}

Under our proposed scheme all MBMS users will report their measured CQI value to Node-B at the time of a call setup. Node-B after considering the distribution of UEs based on their reported CQIs decides the optimal no. of groups and range of each group. The groups are then mapped to different MCS. This information is then passed on to all the UEs through one control channel i.e. Downlink Shared Control Channel (DSCCH). Each UE is required to decode $\mathrm{DSCCH}$ before attempting to decode upcoming TTI High Speed Downlink Shared Channel (HS-DSCH) code. UEs will keep record of its CQI in each TTI and uses it to decode appropriate group in the upcoming TTI. UE receiving CQI 1 or 0 is considered to be in outage for this transmission. If, for example, a UE receives CQI 5 in current TTI then it will decode group 2 stream in the upcoming TTI. Since selection of group is 1 TTI delayed from when the CQI was received, moving to lower CQI group will involve loss of packet in at least 1 TTI. The number of groups and each group CQI range are dynamically managed at Node-B with minimum required Uplink signalling. Each UE keeps track of its own CQI and reports back its CQI to Node-B only after it receives a CQI that falls into a different group for 3 consecutive TTIs. Node-B continuously calculates the optimal group sizes and changes the Enhancement layer mapping to suit the new group range.

The scheme uses the Adaptive Modulation and Coding (AMC) mechanism of the HSDPA standard at the group level rather than at the individual user level. The grouping mechanism improves the overall QoS of the system because groups with higher CQI values will not be constrained by the lower CQI UEs thus improving the total QoS as well as the coverage area. The number of groups can be optimised based on the user requirements and application data rates. The approach is scalable and requires minimum signalling on the Uplink.

\section{Simulation Modelling}

A discrete-time event-driven simulation model was developed based on the OPNET Modeler package to analyse the performance of the proposed scheme. The transmission channel model includes path loss, shadow and multipath fading models. The path loss was modelled using COST 231 Hata Model. The shadow fading was modelled using the lognormal distribution and the correlation distance. The multipath fading was modelled using the $3 \mathrm{GPP}$ Pedestrian A ( $3 \mathrm{~km} / \mathrm{h})$ model using the tapped delay line generating random number of taps and combing them to generate the loss in each TTI. The Signal to Interference and Noise Ratio (SINR) value was calculated at the output of the receiver using the equation 1.

$$
\operatorname{SINR}=\frac{P_{H S-D S C H}}{P_{o w n}\left[(1-\alpha)+G^{-1}\right]}
$$

Where $P_{H S-D S C H}$ is power allocated to the HS-DSCH, $P_{O w n}$ is total Node-B power, $\alpha$ is the Orthogonality factor and $\mathrm{G}$ is the geometry factor given by the equation 2 .

$$
G=\frac{P_{\text {own }}}{P_{\text {other }}+N}
$$

$P_{\text {other }}$ is the total interference generated by other cells and $N$ is the thermal Noise. All 50 UE move in random direction at $3 \mathrm{Km} / \mathrm{h}$ speed using Random Waypoint Mobility profile in OPNET.

TABLE II. HSDPA SINR Targets for different CQI

\begin{tabular}{|c|c|c|}
\hline CQI & Bit Rate (Kbps) & SINR (dB) \\
\hline 1 & 68.5 & -8.88 \\
\hline 2 & 86.5 & -7.77 \\
\hline 3 & 116.5 & -6.33 \\
\hline 4 & 158 & -4.89 \\
\hline 5 & 188.5 & -3.99 \\
\hline 6 & 230.5 & -3.11 \\
\hline 7 & 325 & -1.37 \\
\hline 8 & 396 & -0.51 \\
\hline 9 & 465.5 & 0.19 \\
\hline
\end{tabular}

TABLE III. Simulation Parameters

\begin{tabular}{|c|c|}
\hline Parameter & Value \\
\hline No. of Node-B & 1 \\
\hline Antenna type & Omnidirectional \\
\hline Cell Radius & $933 \mathrm{~m}$ \\
\hline BS Antenna gain + cable loss & $15 \mathrm{~dB}$ \\
\hline BS Total Transmit Power & $12 \mathrm{~W}$ \\
\hline HSDPA Used Power & $7 \mathrm{~W}$ \\
\hline Orthogonality Factor $(\alpha)$ & 0.5 \\
\hline Geometry Factor $(\mathrm{G})$ & $-3-10 \mathrm{~dB}$ \\
\hline Maximum no. of codes & 2 \\
\hline Simulation time & $300 \mathrm{sec}$ \\
\hline MBMS Sessions & 1 \\
\hline Link adaptation Algorithm & Standard AMC \\
\hline Total no. of UEs/cell & 50 \\
\hline
\end{tabular}

An Iterative Solution Coded Modulation Library (ISCML) based on the open source toolbox is used to run the link level simulations in Matlab to obtain the Signal to Interference and Noise Ratio (SINR) values [8]. The simulator takes into account the channel 
conditions, interleaving, channel coding, receiver structure and decoding to predict the Block Error Rate (BLER) of the receiver. These values were derived for MBMS services corresponding to $1 \%$ BLER for different CQI values. Table II lists transmission bit rate and SINR values for different CQI values. Category 7 and 8 UEs were considered for our simulations. Initial simulations were run with 50 UEs in three scenarios. In our simulation we consider that a UE is inside an MBMS coverage area if the UE receives information with a BLER of $1 \%$ or less. Key simulation parameters are listed in the Table III.

\section{Simulation Results}

In this section we analyse various simulation results obtained using the OPNET model. Fig. 1 shows the MBMS coverage distribution areas for different transmission data rates for $933 \mathrm{~m}$ and $500 \mathrm{~m}$ cell sizes. To obtain this result we used a single/nongroup based transmission and run the simulation for different CQI values. As shown on the figure that if we require higher coverage areas for MBMS services then the transmission data rate will be restricted by lowest CQI UEs in the cell. The coverage drops almost linearly with the increasing data rate. The coverage drops to only $12 \%$ at highest achievable data rate of $396 \mathrm{kbps}$ for $933 \mathrm{~m}$ cell size. Same coverage pattern can be observed for a $500 \mathrm{~m}$ cell size. This simulation did not include the HARQ option which could introduce additional delay and a multicast synchronisation problem.

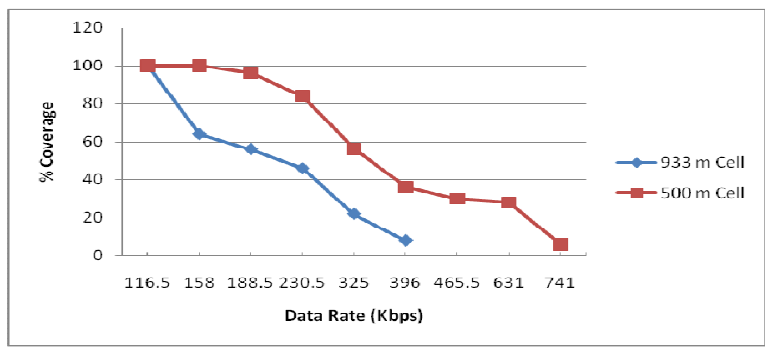

Figure 1. Distribution of coverage areas for non-group based MBMS transmission scheme.

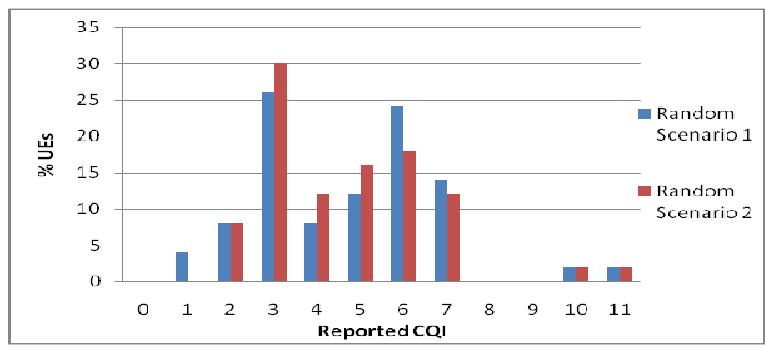

Figure 2. CQI distribution of UEs

Next we run the simulation first with stationary UEs and then with UEs moving in random directions with an average $3 \mathrm{Km} / \mathrm{h}$ speed. The Stationary UE simulation had two scenarios in which stationary
UEs are randomly distributed over the entire cell. Linear congruential pseudorandom number generator is used to derive the random position coordinates of UEs from the BS with different seed values both of random distribution scenario 1 and 2. Fig. 2 shows the distribution of Reported CQI in both scenarios. Only 8\% UEs have reported CQI 2 in both scenarios and rest are reporting higher values. Random Scenario has $4 \%$ UEs reporting CQI 1. A multicasting scheme transmitting MBMS content in the $\mathrm{p}-\mathrm{t}-\mathrm{m}$ mode based on minimum reported CQI of

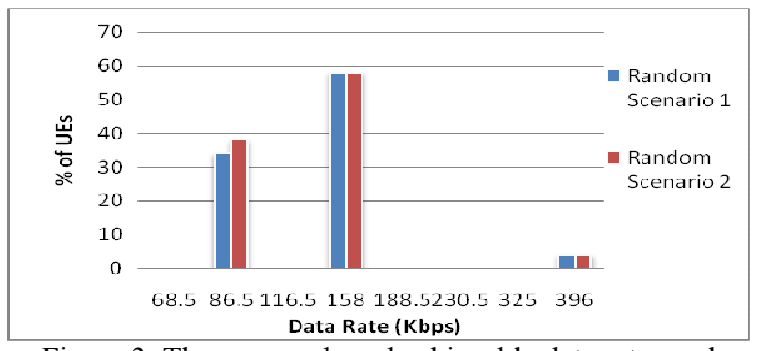

Figure 3. Three group based achievable data rates and MBMS coverage areas

all the UE will achieve very low throughput and therefore low quality video. $96 \%$ and $100 \%$ UEs in Random scenario 1 and 2 respectively, will only be able to decode Base layer if non-group approach is used. Fig. 3 shows that if we divide UEs into groups based on their reported CQI, 58\% UEs in both scenarios will be able to decode Base and $1^{\text {st }}$ Enhancement layer giving them a much better quality video. It can also be observed that $4 \%$ UEs can receive all three layers.

Results shown in Fig. 4-7 are taken from second run of the simulation with UEs randomly moving within a cell. Fig. 4 shows the distribution of reported CQI by UEs at the start, $120 \mathrm{sec}$ and $240 \mathrm{sec}$ time interval of our $300 \mathrm{sec}$ simulation. A single group based scheme can at maximum allow all the UEs to decode the Base layer only. It is clearly visible from the distribution that the largest no. of UEs have reported CQI between 4-6 where as the $2^{\text {nd }}$ largest group lies between CQI 2-3. An average of 5$10 \%$ UEs have reported CQI 8 or higher. An interesting observation here is that if we compare the CQI distribution of mobile UEs with that of stationary ones, the pattern of distribution is almost identical as shown in Fig 2. and Fig 4. This signifies the need of a scheme which can accommodate variable channel quality users and deliver them the better quality multimedia content if appropriate channels are allocated.

Using the group based transmission scheme a Node-B upon connection setup determines the number of UEs in a group and their reported CQI. It then decides the optimal group range and then map that range to Base layer and matching Enhancement layers. Table I. shows the mapping for this simulation. Although the mapping can change over 
time based on UEs switching the groups, for this simulation we have kept this mapping constant for the duration of simulation.

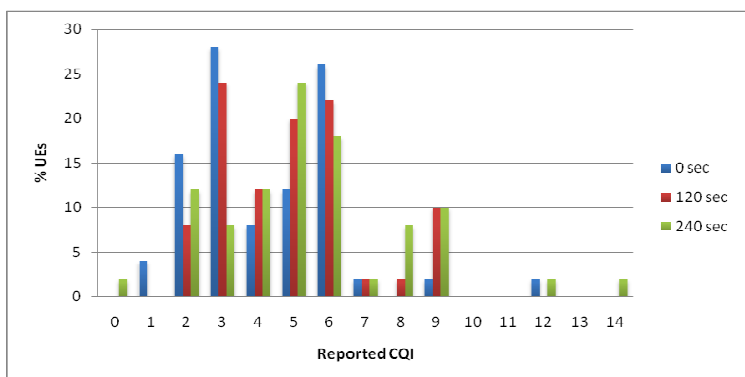

Figure 4. UE CQI distribution at different Simulation Time

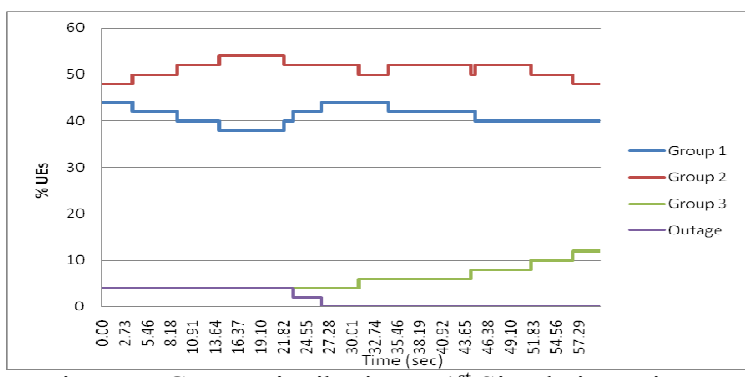

Figure 5. Group Distribution at $1^{\text {st }}$ Simulation Minute

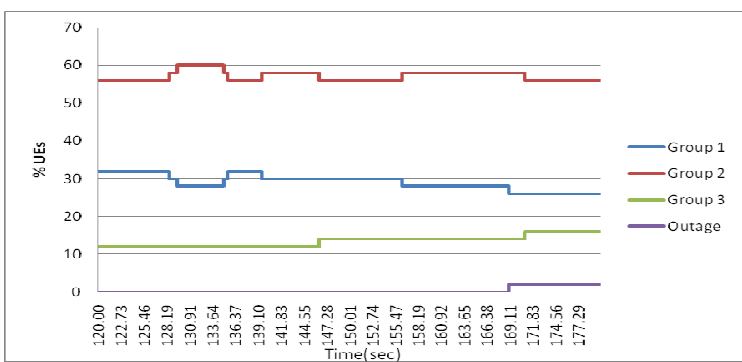

Figure 6. Group Distribution at $3^{\text {rd }}$ Simulation Minute

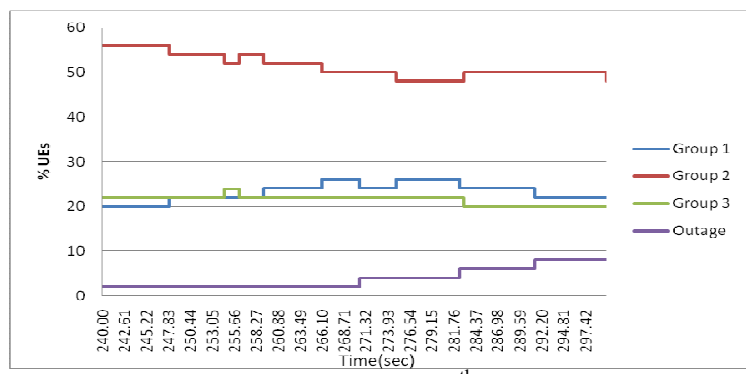

Figure 7. Group Distribution at $5^{\text {th }}$ Simulation Minute

Fig 5-7 shows the \% of UEs that are member of each group over the $1^{\text {st }}, 3^{\text {rd }}$ and $5^{\text {th }}$ minute of the simulation. It can be seen that Group 2 accounts for an average of $58 \%$ UEs allowing a large proportion of UEs to decode Base and 1 Enhancement layer. Group 1 stayed between $30-40 \%$ by the middle of simulation but started to fall to about $20 \%$ near the end of simulation. Group 3, receiving the highest quality content, shows an increasing trend accounting for $20 \%$ of total UEs by the simulation end. Outage i.e. UEs reporting CQI 1 or less, remained less that $5 \%$ on average during $300 \mathrm{sec}$ simulation.
Simulation results clearly indicate that signifcant QoS improvments can be achieved for MBMS services when the group based transmission schemes are used. As mentioned earlier that the proposed scheme does not require too much feedback to manage these groups. The UEs only requires to report back their CQI if it falls outside their current group for multiple TTIs. The scalable video coder only requires to encode the video once into multiple layers instead of encoding the same video multiple times into different quality streams. This effectively lowers the processing resoure requirement of encoder. Node-B dynamically decides the no. of enhancement layers to be sent alongwith the base layer. The decision is made based on UEs distribution, existing group membership and range, enhancement layer availability for corresponding data rate and available no. of orthogonal codes. The proposed scheme not only introduces higher QoS, higher QoE and lower signalling rate but also offers scalability feature and can serve large no. of users.

\section{Conclusion}

The paper presented an efficient group based MBMS performance enhancement scheme. Simulation results show that the proposed scheme offer signification improvements of MBMS service QoS when combined with a scalable or a hierarchical video coder. The dynamic nature of group management ensures best quality content to be delivered to each user with minimal air interface resources. The proposed algorithm can also support terminal mobilities without reducing the overall QoS in a cell.

\section{References}

[1] "MBMS Architecture and functional description, Technical realization stage 2," 3GPP TSG TS23.246, Rev.6.1.0, Dec 2003.

[2] S. Parkvall et al. "Evolving 3G Mobile Systems: Broadband and Broadcast Services in WCDMA," in IEEE Communication Magazine.vol.44,2006,pp.68-74.

[3] "High Speed Downlin Packet Access (HSDPA) Stage 2," 3GPP TS 25.308 v5.4.0 Release 6, March 2004.

[4] A. Asif, U. T. Nguyen, and G. Xu, "Scalable Video Multicast over MANETs," in 8th IEEE Workshop on Multimedia Signal Processing, 2006, pp. 403-408.

[5] F. Leitao and A. Correia, "HSDPA Delivering MBMS Video Streaming," in 8th International Symposium on Wireless Personal Multimedia Communications, Aalborg, Denmark, 2005, pp. 1538 - 1542.

[6] A. M. C., J. C. M. Silva, N. M. B. Souto, L. A. C. Silva, A. B. Boal, and A. B. Soares, "Multi-Resolution Broadcast/Multicast Systems for MBMS," IEEE Transactions on Broadcasting, vol. 53, pp. 224 - 234, March 2007.

[7] V. Vartiainen and J. Kurjenniemi, "Point-to-Multipoint Multimedia Broadcast Multicast Service (MBMS) Perfromance Over HSDPA," in PIMRC07, Greece, 07.

[8] http://iterativesolutions.com/Matlab.htm." vol. 2007. 